\title{
Line-profile Formula in the Carbon Nanotubes by Electron Spin Resonance
}

\author{
Jung-Il Park ${ }^{1}$ and Haeng-Ki Lee ${ }^{2^{*}}$ \\ ${ }^{1}$ Nano Application Laboratory, Department of Physics, Kyungpook National University, Daegu 702-701, Korea \\ ${ }^{2}$ Department of Radiotechnology, Suseong College, Daegu 706-711, Korea \\ (Received December 29, 2011; Revised May 26, 2012; Accepted June 08, 2012)
}

\begin{abstract}
The line-width of carbon nanotubes (CNTs) was studied as a function of the temperature at a frequency of $9.49 \mathrm{GHz}$ in the presence of external electromagnetic radiation. The relative frequency dependence of the absorption power is obtained with the projection operator technique (POT) proposed by Kawabata. The line-width increased as the temperature increased in the hightemperature region $(\mathrm{T}>200 \mathrm{~K})$. The scattering is little affected in the lowtemperature region $(\mathrm{T}<200 \mathrm{~K})$ because there is no correlation between the resonance field and the Fermi-Dirac distribution function. Thus, the present technique is considered to be more convenient to explain the resonant system as in the case of other optical transition problems.
\end{abstract}

Keywords: ESR, CNTs, Projection operator, Line-profile, Line-widths,

\section{INTRODUCTION}

Electron spin resonance (ESR) spectroscopy has been used to assess the quality of carbon nanotube, CNT. ${ }^{1}$ In ESR experiments, one applies a static magnetic field and measures the absorption power of electromagnetic radiation polarized perpendicular to the field direction. In the absence of SU(2) spin symmetry breaking terms in the system Hamiltonian, the absorption power is then simply

Department of Radiotechnology, Suseong College, 15, Dalgubeol daero-528gil, Suseong-gu, 706-711, Korea Tel: +82.53.749.7104 Fax: +82.53.749.7103 email:leeki@sc.ac.kr Journal of the Korean Magnetic Resonance Society 2012 June; 16(1): 11-21 http://dx.doi.org/10.6564/JKMRS.2012.16.1.011 
a delta peak at the Zeeman energy. Since spin-orbit coupling (SOC) are generally the leading terms breaking the $\mathrm{SU}(2)$ invariance, deviations in the absorption power from delta peak, e.g., shifts or broadenings, are directly connected to these coupling. Initially, it was assumed that a SOC in CNTs, due to $p_{z}$ electrons, would lead to a long spin relaxation compared with typical semiconductors. However, numerous experimental measurements of spin transport and ESR in CNTs present some different results for the magnitude of the spin relaxation. ${ }^{2,3}$

From a theoretical point of view, the studies performed thus far on a resonant system in the presence of electromagnetic radiation have usually been based on the following methodologies: Green's function approach, Feynmann's path integral approach, the Wigner representation approach, and the projection operator technique (POT). There have been numerous methods for the calculation of resonance line-widths. Among the above mentioned methods, we focus on the POT of Kawabata. ${ }^{4}$ By using this method, we succeeded in formulating a response theory, ${ }^{5-8}$ which includes the Kubo ${ }^{9}$ formalism as the lowest-order approximation.

In the present work, we calculate the line-width with the same proposition that Kawabata used and compare experimental data of Salvetat et al. ${ }^{10}$ Through numerical calculations, we analyze the relative frequency dependence of the absorption power and the temperature dependence of the linewidth at a frequency of $9.49 \mathrm{GHz}$ in the presence of external radiation. Finally, we discuss the results and draw conclusions. 


\section{SYSTEM}

We begin with a few essential definitions concerning CNTs. The CNT is conveniently imagined as a spiral graphite sheet (graphene) rolled along the chiral vector $\vec{C}_{h}$ as below (see Fig. 1)

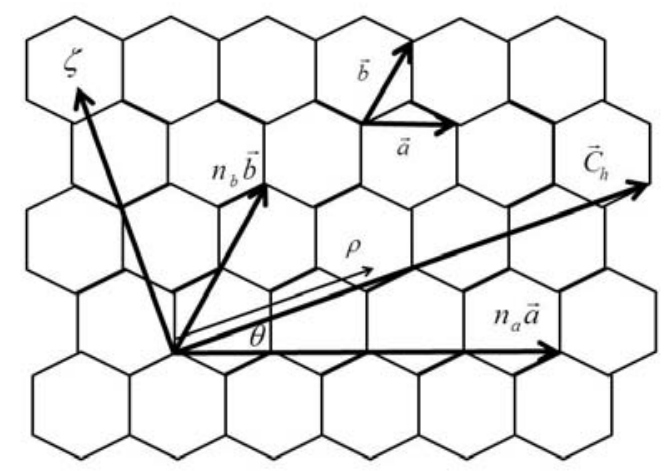

Figure 1. The lattice structure of graphene.

$$
\vec{C}_{h}=n_{a} \vec{a}+n_{b} \vec{b},
$$

where $\vec{a}=a_{c c}(1,0)$ and $\vec{b}=a_{c c}(1 / 2, \sqrt{3} / 2)$ are the graphene lattice unit vectors with $a_{c c}=0.246 \mathrm{~nm}$ and $n_{a}$ and $n_{b}$ are integers, which characterize the geometry of a particular CNT. The slop of $\vec{C}_{h}$ is defined by

$$
\theta=\tan ^{-1}\left[\frac{\sqrt{3} n_{b}}{2 n_{a}+n_{b}}\right],
$$


and the diameter of the CNT is given by $\left|\vec{C}_{h}\right|=\sqrt{3} a_{c c}\left(n_{a}+n_{b}+n_{a} n_{b}\right)^{1 / 2}, d=\left|\vec{C}_{h}\right| / \pi$. The graphene two-valley band structure projects onto the CNT one so that in the vicinity of each valley the Hamiltonian takes the form: ${ }^{12}$

$$
H_{\lambda}=H_{z}+\hbar v_{F}\left(\lambda \kappa_{m} \sigma_{1}+k \sigma_{2}\right),
$$

where the Fermi velocity is $v_{F}=3 \times 10^{5} \mathrm{~m} / \mathrm{s}, \sigma_{1}$ and $\sigma_{2}$ are Pauli spin matrices and, $k$ is the wave vector of electron. The energy band structure of graphene are located at the $K$ and $K^{\prime}$ corner points of the first Brillouin zone. Which will be labeled by $\lambda=+1,-1$ respectively. In the reference frame $(\xi, \eta, \zeta)$, the Zeeman Hamiltonian takes the form $H_{z}=\hbar \omega_{z}\left(\sin \alpha S_{\xi}+\cos \alpha S_{\zeta}\right)$, $\hbar \omega_{z}=g_{e} \mu_{B} B$ ( $g_{e}=2$ and $\mu_{B}$ is the Bohr magneton) so that the frequency of spin procession is given by

$$
\omega_{\lambda}=\sqrt{\left(\omega_{z} \sin \alpha\right)^{2}+\left[\omega_{z} \cos \alpha+2 \lambda\left(\Lambda_{0}+\Lambda_{1}\left\langle\sigma_{1}\right\rangle\right) / \hbar\right]^{2}}
$$

$\left|\Phi_{k,+m, \lambda}^{C N T}\right\rangle$ is an eigenstates of $H_{\lambda}$ for an spin-up electron; and $\left|\Phi_{k,-m, \lambda}^{C N T}\right\rangle$ for a down-spin electron. Then, $\left|\Phi_{k, \pm m, \lambda}^{C N T}\right\rangle$ satisfies the Schrödinger equation as below

$$
H_{\lambda}\left|\Phi_{k, \pm m, \lambda}^{C N T}\right\rangle=\left( \pm \hbar v_{F} \sqrt{\kappa_{m, \lambda}^{2}+k^{2}}+\hbar \omega_{\lambda}\right)\left|\Phi_{k, \pm m, \lambda}^{C N T}\right\rangle
$$

where $E_{ \pm m}= \pm \hbar v_{F} \sqrt{\kappa_{m, \lambda}^{2}+k^{2}}+\hbar \omega_{\lambda}$ is the CNT electronic spectrum. When a SOC is incorporated in the Hamiltonian $H_{\lambda}$, the SOC mediated by CNT curvature which take the simplest form in CNT coordinate as: 


$$
H_{s o}=\Lambda_{0} \lambda \sigma_{0} 2 S_{\zeta}+\Lambda_{1} \lambda \sigma_{1} 2 S_{\zeta}-i \Lambda_{2} \sigma_{2}\left(S_{+} e^{i \varphi}-S_{-} e^{-i \varphi}\right)
$$

where $\sigma_{0}$ is identical matrix, $S_{ \pm}=S_{\xi} \pm i S_{\eta}, \varphi=2 \rho / d, 0<\rho<\left|\vec{C}_{h}\right|$. The electron spin operators $\sigma_{ \pm}=\sigma_{x} \pm i \sigma_{y}$ are defined as $\sigma_{+}=2 \sum_{m} a_{m+}^{+} a_{m-}, \sigma_{-}=2 \sum_{m} a_{m-}^{+} a_{m+}$. In Eq. (6), the SO constants are proportional to CNT curvature, $\Lambda_{0}=\left(\delta_{0} / d\right) \cos 3 \theta, \Lambda_{1,2}=\delta_{1,2} / d$, $\delta_{1}=-0.19 \mathrm{meV} \cdot \mathrm{nm}, \delta_{0} / \delta_{1}=4.5, \delta_{2} / \delta_{1}=-1.4, \alpha=\pi / 2$. We take into account that $\left\langle k, m, \lambda\left|\sigma_{0}\right| k, m, \lambda\right\rangle=1, \quad\left\langle k, m, \lambda\left|\sigma_{2} e^{ \pm i \varphi}\right| k, m, \lambda\right\rangle=0, \quad$ and $\left\langle k, m, \lambda\left|\sigma_{1}\right| k, m, \lambda\right\rangle= \pm \lambda \kappa_{m, \lambda} / \sqrt{k^{2}+\kappa_{m}^{2}}$. Here $+(-)$ corresponds to conduction (valence) band.

\section{PROJECTION OPERATOR TECHNIQUE}

When a polarized electromagnetic radiation with angular frequency $\omega$ is applied along the $z$-axis, the absorption power $P_{a b s}(\omega)$ delivered to the system is given by

$$
P_{a b s}(\omega)=\left(H_{0}^{2} / 2\right) \operatorname{Re}\left[\chi_{+-}(\omega)\right] \text {, }
$$

here "Re" means "the real part of" and the electron spin susceptibility (ESS) $\chi_{+-}(\omega)$ is given, in the Kubo formalism, as

$$
\chi_{+-}(\omega)=\frac{1-e^{-\beta \omega_{z}}}{\omega_{z}} \sum_{ \pm m} f\left(E_{ \pm m}\right)\left[1-f\left(E_{ \pm m}+\hbar \omega_{z}\right)\right]
$$




$$
\times\left\langle k,+m, \lambda\left|\sigma_{-}\right| k,-m, \lambda\right\rangle\left\langle k,-m, \lambda\left|\sigma_{-}\right| k,+m, \lambda\right\rangle\left\langle\Xi_{+-}^{e p r}(\omega)\right\rangle,
$$

where $f\left(E_{ \pm m}\right)$ is the Fermi-Dirac distribution function for the electron state $\left|\Phi_{k, \pm m, \lambda}^{C N T}\right\rangle$. It is convenient to write the expression of $\left\langle k,+m, \lambda\left|\sigma_{+}(t)\right| k,-m, \lambda\right\rangle$ in the second quantization formalism as $\left\langle k,+m, \lambda\left|\sigma_{+}(t)\right| k,-m, \lambda\right\rangle=\operatorname{Tr}^{(e)}\left\{a_{-m}^{+} a_{+m} \sigma_{+}(t)\right\}$, where $\sigma_{+}(t)$ on the right hand side is a second quantized operator. Following Kawabata, ${ }^{4}$ we define two projection operators $P_{+-}$and $Q_{+-}$as

$$
P_{+-} Y \equiv \frac{\left(X_{+m-m}, Y\right)}{\left(X_{+m-m}, \sigma_{+}\right)} \sigma_{+}, Q_{+-} Y \equiv \frac{\left(Y, \sigma_{+}\right)}{\left(X_{+m-m}, \sigma_{+}\right)} X_{+m-m},
$$

with $X_{+m-m}=a_{-m}^{+} a_{+m}$. We easily see that $P_{+-}$and $Q_{+-}$satisfy the condition imposed on projection $\quad$ operators, $\quad P_{+-}^{2}=P_{+-} \quad, \quad Q_{+-}^{2}=Q_{+-} \quad$ and $\left(Q_{+-} X,\left(1-P_{+-}\right) Y\right)=\left(\left(1-P_{+-}\right) X, P_{+-} Y\right)=0$. We consider the equation of motion as below

$$
\Xi_{+-}(t) \equiv \frac{\left(X_{+m-m}, \sigma_{+}(t)\right)}{\left(X_{+m-m}, \sigma_{+}\right)} .
$$

We separate $\tilde{X}_{+m-m}$ into two parts, parallel and orthogonal to $\tilde{X}_{+m-m}$. Then we obtain from Eq. (10)

$$
\begin{aligned}
& \frac{d \Xi_{+-}(t)}{d t}=-\frac{\left(\exp (-i L t) \tilde{X}_{+m-m}, \sigma_{+}\right)}{\left(X_{+m-m}, \sigma_{+}\right)}=i \omega_{0} \Xi_{+-}(t)-\frac{\left(\left(1-K_{+m-m}\right) X_{+m-m}, \sigma_{+}(t)\right)}{\left(X_{+m-m}, \sigma_{+}\right)}, \\
& i \omega_{0}=-\frac{\left(\tilde{X}_{+m-m}, \sigma_{+}\right)}{\left(X_{+m-m}, \sigma_{+}\right)}=\frac{\left(X_{+m-m}, \tilde{\sigma}_{+}\right)}{\left(X_{+m-m}, \sigma_{+}\right)},
\end{aligned}
$$

where $\tilde{X}_{+m-m}=i L X_{+m-m}$ and $K_{+m-m}=\left(1-Q_{+-}\right) X_{+m-m}$. 
Next we separate $\sigma_{+}(t)$ into two parts, parallel and orthogonal to $\sigma_{+}$, and using the useful relations $L_{f} \sigma_{+}=\omega_{0} \sigma_{+}, Q_{+-} L_{f} \sigma_{+}=0,\left(L_{f} Q_{+-} X\right)_{+m-m} 0$, since $P_{+-} \sigma_{+}=\sigma_{+}$, from Eq. (11) it follows that $K_{+m-m}$ and $\sigma_{+}$are orthogonal to each other, i.e., $\left(Q_{+-}, \sigma_{+}\right)=0$. Then we obtain

$$
\frac{d \Xi_{+-}^{e p r}(t)}{d t}=i \omega_{0} \Xi_{+-}^{e p r}(t)-\int_{0}^{t} d \omega \Xi_{+-}^{e p r}(\omega) \Gamma_{+-}^{e p r}(t-\omega),
$$

where $\Gamma_{+-}^{e p r}(t)=\left(K_{+m-m}, R_{+m-m}(t)\right) /\left(X_{+m-m}, \sigma_{+}\right)$. We obtain the ESS as below

$$
\chi_{+-}(\omega)=\sum_{ \pm m} \frac{f\left(E_{+m}\right)-f\left(E_{-m}\right)}{E_{+m}-E_{-m}} \frac{\left|\left(\sigma_{+}\right)_{+m-m}\right|^{2}}{i \hbar\left(\omega-\omega_{0}\right)-i \Gamma_{+-}^{e p r}(\omega)},
$$

We see the coherent scattering factor $\Gamma$, call the line-profile formula, gives characteristics of the system because it is affected by the resonance mechanism. The lowest-order approximation of the line-profile is given as follows:

$$
\begin{aligned}
& \Gamma_{+-}^{e p r}(\omega)=\sum_{\nu \neq \mu} \sum_{\Delta m \neq \pm 1} \frac{1}{\left(\sigma_{+}\right)_{+m-m}}\left\{\left(1+f\left(E_{ \pm m}\right)\right)\left[\sum_{j=1}^{2} \frac{\left(\sigma_{+}\right)_{-m+m} A j}{\hbar \omega-\varepsilon j^{+}}-\sum_{j=3}^{4} \frac{\left(\sigma_{+}\right)_{+m-m} A j}{\hbar \omega-\varepsilon j^{+}}\right]\right. \\
& \left.+f\left(E_{ \pm m}\right)\left[\sum_{j=1}^{2} \frac{\left(\sigma_{+}\right)_{-m+m} A j^{*}}{\hbar \omega-\varepsilon j^{-}}-\sum_{j=3}^{4} \frac{\left(\sigma_{+}\right)_{+m-m} A j^{*}}{\hbar \omega-\varepsilon j^{-}}\right]\right] .
\end{aligned}
$$

Eq. (14) is similar to Sawaki's result, ${ }^{13}$ which is based on the Stark ladder representation. However, our formula uses more terms for expressing the Fermi-Dirac distribution. We consider the term $\Gamma_{+-}^{e p r}(\omega) \equiv i S_{+-}^{e p r}(\omega)+W_{+-}^{e p r}(\omega)$, where the line-shift spectra is $S_{+-}^{e p r}(\omega)=\operatorname{Im}\left[\Gamma_{+-}^{e p r}(\omega)\right]$, and 
the line-width is $W_{+-}^{e p r}(\omega)=\operatorname{Re}\left[\Gamma_{+-}^{e p r}(\omega)\right]$. We apply this approximate formula to the real system for demonstration, we obtain absorption power as:

$$
P_{a b s}\left(\omega-\omega_{0}\right) \propto \frac{1}{\omega_{z}} \int_{-\infty}^{+\infty} d E_{ \pm m} \frac{W_{+-}^{e p r}(\omega)\left[f\left(E_{-m}\right)-f\left(E_{+m}\right)\right]}{\left[\omega-\omega_{0}-S_{+-}^{e p r}(\omega)\right]^{2}+\left[W_{+-}^{e p r}(\omega)\right]^{2}}
$$

The absorption power is described by the Lorentzian form. Fig. 2 shows the relative frequency dependence of the absorption power, for the spectrum of the CNT at 150, 200, 250, and $300 \mathrm{~K}$. From the absorption power, we can see the broadening effect near the resonance peak, which exhibits increase as the temperature increases. In Fig. 3, the temperature dependence of line-width compare with experimental data of Salvetat et al. ${ }^{10}$

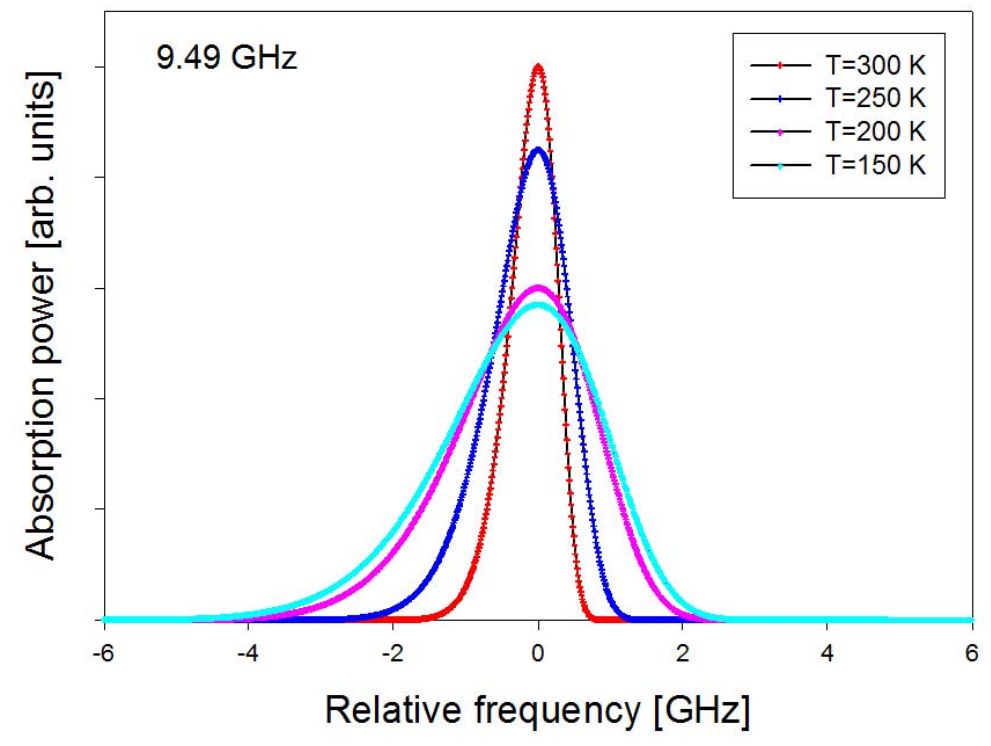

Figure 2. The Relative frequency dependence of the absorption power $P_{a b s}\left(\omega-\omega_{0}\right)$ curves of EPR at 150, 200, 250, and $300 \mathrm{~K}$ in the CNT at a frequency of $9.49 \mathrm{GHz}$. 


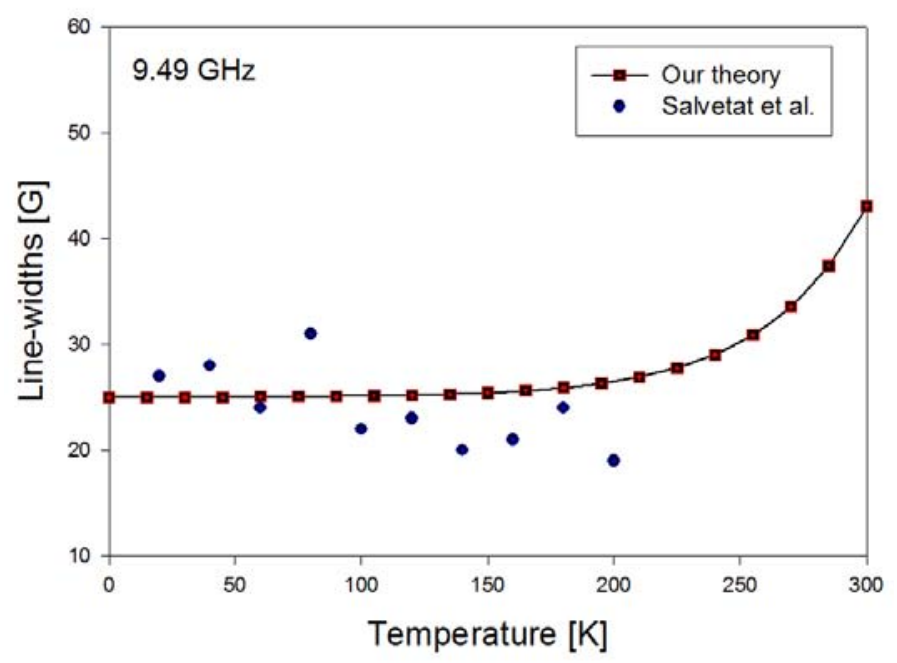

Figure 3. The temperature dependence of the line-width in the presence of an external radiation at a frequency of $9.49 \mathrm{GHz}$.

\section{CONCLUSIONS}

Using Kawabata's POT, in the CNT, we derived the line-profile formulas that show the relative frequency dependence of the absorption power and the temperature dependence of the line-width. We strictly used the commutation relation of annihilation and creation operators with using the Kubo identity. From the absorption power, on the order of 150, 200, 250, and $300 \mathrm{~K}$, we can see the broadening effect of absorption power near the resonance peak, which exhibits a small increase as the temperature increase in the high-temperature region $(\mathrm{T}>200 \mathrm{~K})$. The line-width (the inverse of the spin relaxation) monotonically increases with increasing temperature $(T>200 \mathrm{~K})$ due to the 
interaction of electrons with acoustic phonons. The ESR scattering effect is little affected in lowtemperature region $(\mathrm{T}<200 \mathrm{~K})$ because there is no correlation between the resonance field and FermiDirac distribution function. The line-width obtained with POT agrees with the experimental result in low-temperature region. We conclude that the calculation process presented in this work is useful for studying the ESR in the CNT.

\section{Acknowledgment}

This research was supported by Basic Science Research Program through the National Foundation of Korea (NRF) funded by the Ministry of Education, Science and Technology (NRF2011-0027409). Also we thank the Suseong College Research Fund.

\section{REFERENCES}

1. Bachtold, P. Hadley, T. Nakanishi, C. Dekker, Science 294, 1317 (2001).

2. O. Chauvet, L. Forro, W. Bacsa, D. Ugarte, B. Doudin, Walt A. de Heer, Phys. Rev. B52, R6963 (1995).

3. L. Forro et al, in "Science and Application of Nanotubes" (Tomanek, Enbody, Ed.), Kluwer Academic Publishers, New York, 2000.

4. A. Kawabata, J. Phys. Soc. Jpn. 29, 902 (1970).

5. J. Y. Sug, S. D. Choi, Phys. Rev. E55, 314 (1996).

6. J. Y. Sug, S. G. Jo, S. D. Choi, Phys. Rev. B64, 235210 (2001).

7. J. I. Park, J. Y. Sug, H. R. Lee, J. Kor. Phys. Soc. 53, 776 (2008).

8. J. I. Park, H. K. Lee, H. R. Lee, J. Magnetics 16, 108 (2011).

9. R. Kubo, J. Phys. Soc. Jpn. 12, 570 (1957). 
10. J. P. Salvetat, T. Feher, L. Forro, Phys. Rev. B72, 75440 (2005).

11. A. Thess, R. Lee, P. Nikolaev, H. Dai, P. Petit, Science 273, 483 (1996).

12. T. Ando, J. Phys. Soc. Jpn 74, 777 (2005).

13. N. Sawaki, J. Phys. C16, 4611 (1983). 